\title{
Gestión de materiales y residuos radiactivos en la Universidad de Tübingen, Alemania
}

\author{
Tübingen University Radioactive Material \\ and Waste Management
}

Jose Carlos Mora-Barrantes'

Roy Zamora-Sequeira²

David Benavides-Ramírez ${ }^{3}$

Thomas Haug ${ }^{4}$

Fecha de recepción: 28 de julio del 2013

Fecha de aprobación: 19 de noviembre del 2013

Mora-Barrantes, j; Zamora-Sequeira, R; Benavides-Ramírez, D; Haug, T. Gestión de materiales y residuos radiactivos en la Universidad de Tübingen, Alemania. Tecnología en Marcha. Vol. 27, № 2. Pág 22-31

Químico Industrial, Universidad Nacional, Costa Rica. Teléfono: 22773403. Correo electrónico: jose.mora.barrantes@una.cr.

2 Químico Industrial. Instituto Nacional de Aprendizaje, Costa Rica. Teléfono: 22902024. Correo electrónico: zamoraroy@yahoo.com.

3 Químico Industrial. Instituto Tecnológico de Costa Rica, Costa Rica. Teléfono: 89936448. Correo electrónico: dbenavides@itcr.ac.cr.

4 Químico Físico. University of Tübingen, Alemania. Teléfono: +49 707| 29-77874. Correo elctrónico: thomas.haug@uni-tuebingen.de. 


\section{Palabras clave}

Material radiactivo; residuos radiactivos; salud; ambiente; gestión adecuada.

\section{Resumen}

Un material radiactivo es toda sustancia o átomo que emite radiaciones ionizantes y no ionizantes; la radiactividad puede ser natural, artificial o inducida. La radiactividad se utiliza en el mundo en ramas de la ciencia como ecología, medicina, agricultura, industria, biología y química. En Costa Rica se usan materiales radiactivos en campos de aplicación industrial, médica y universitaria. Las fuentes radiactivas van desde las de muy baja radiactividad hasta las de muy alta actividad, por ejemplo, $\mathrm{Am}^{241}$ y $\mathrm{Ra}^{226}$, utilizadas en centros universitarios costarricenses. No obstante, si los materiales radiactivos no se manipulan correctamente, pueden tener efectos negativos en la salud y el medio ambiente, por lo tanto es necesario tomar medidas preventivas y protectoras. Costa Rica regula el uso de materiales radiactivos mediante el Decreto Ejecutivo $N^{\circ}$ 24037-S., sin embargo, no existe formalmente un plan nacional de manejo y eso limita su gestión en las instituciones donde se utilizan y por ende el tratamiento y disposición final de los residuos. El presente trabajo de investigación analiza la gestión en materiales radiactivos que realiza el Laboratorio de Isotopos y Protección Radiológica de la Universidad de Tübingen, Alemania, mediante la determinación y análisis de diferentes acciones y datos relacionados con I) aspectos administrativos (manuales, protocolos, normativas) y 2) aspectos técnico-operativos (control de la contaminación, infraestructura, equipos y materiales de seguridad, tratamiento de residuos). La información se recopiló y analizó mediante herramientas como cuestionarios, listas de chequeo, entrevistas, visitas de inspección, sesiones de trabajo y revisión de documentos.

\section{Keywords}

Radioactive material; radioactive waste; health; environment; adequate management.

\begin{abstract}
Radioactivity is the process by which a nucleus of an unstable atom loses energy by emitting particles of ionizing and no ionizing radiation. Radioactivity could be natural or induced. Worldwide, the radioactive materials have many applications in science fields like; ecology, medicine, agriculture, industrial, biology and chemistry. In Costa Rica radioactive materials are use in hospitals, industries and universities. Sources used presents low and high activity level, for example the ones used in universities are $\mathrm{Am}^{241}$ y $\mathrm{Ra}^{226}$. However in order to protect human health and environment, radioactive materials must be managed correctly. Costa Rica radioactive material management is base on regulation 24037-S. Nevertheless, there is no national plan or strategies to manage the materials in a correct form. There are still limitations for users to manipulate, treat and dispose radioactive materials. This work analyzed the radioactive material management at Isotope Laboratory \& Radiation Protection, University of Tübingen. Two main aspects were evaluated; I - Administrative Aspects (manuals, protocols and regulation) and 2-Technical-operatives Aspects (pollution control, facilities, equipment and waste management). The information were collected and analyzed by using the following tools; questionnaire, check list, interview, site inspection, work sessions and documents revision.
\end{abstract}




\section{Introducción}

Un material radiactivo es toda sustancia o átomo que emite radiaciones ionizantes y no ionizantes; la radiactividad puede ser natural 0 artificial (Greenberg, 2009). La radiación ionizante posee la energía suficiente para ionizar la materia, desplazando los electrones de sus órbitas, la no ionizante no tiene esta capacidad (McLintock, 1996). Debido a la carga positiva de los protones, estos están constantemente rechazándose unos a otros; la mayoría se mantienen unidos en una situación estable, pero algunas combinaciones de protones y neutrones no pueden mantener el equilibrio y cambian (decaen) espontáneamente hasta alcanzar una forma más estable liberando ciertas partículas. Estos cambios se conocen como radiactividad y esos átomos son «isotopos radiactivos». Para alcanzar su forma más estable, se debe liberar algo de la energía del átomo y es esta energía la que se conoce como «radiación». A los átomos, o "nucleidos", que decaen o se transforman en otros nucleidos se les puede denominar "radionucleidos". Aproximadamente hay 280 nucleidos estables pero la gran mayoría son inestables o "radiactivos" (Berlin \& Stanton, 1989).

La propiedad de los isotopos de emitir radiación se ha convertido en una herramienta útil y necesaria en las diferentes ramas de la ciencia para análisis de elementos trazas, procesos físicos, químicos, biológicos y médicos, como fuentes intensas de radiación en radiografías industriales y medicina nuclear, en la esterilización de material quirúrgico desechable, productos químicos y biológicos, etc. (OECD, 20 I 0, OIEA, 2003). No obstante, los materiales radiactivos tienen efectos importantes en el medio ambiente y en la salud de las personas si no se manipulan de forma segura y correcta (OIEA, 2009, Simon, 2009). Las radiaciones ionizantes pueden romper los enlaces entre los átomos dentro de las moléculas de las células vivas, pudiendo cambiar la naturaleza de los átomos mismos, con el potencial de ocasionar su muerte o un cambio en su funcionamiento.

Los efectos sobre la salud y el medio ambiente dependen de la reacción de los radionucleidos con la materia y de su vida media (tiempo de desintegración) (Berlin \& Stanton, 1989). Las vidas medias varían desde fracciones de segundo a millones de años y de estas depende en gran medida la cantidad de radiactividad recibida por un cuerpo, la cual se expresa como "dosis absorbida" y la unidad de medida es el "gray" (Gy). Debido a que las diferentes partes del cuerpo son afectadas de manera diferente por la misma cantidad de radiación, se creó un factor de ponderación para calcular el riesgo global para el organismo proveniente de las exposiciones parciales, llamado "Dosis Equivalente Efectiva" (la unidad de medición es sieverts o Sv), que es la suma ponderada de las dosis equivalentes para los órganos individuales y la irradiación total del cuerpo. La dosis equivalente en $S_{v}$ es numéricamente igual a la dosis absorbida en Gy (Schleien, 1992). Según los estándares de la Organización Internacional de Energía Atómica (OIEA), los límites anuales de las dosis para un trabajador ocupacionalmente expuesto son los siguientes (Decreto Ejecutivo, 24037, 1995): I) para la totalidad del organismo referido a cualquier periodo de 12 meses consecutivos es de 20 miliSieven, pudiendo superarse este valor siempre y cuando en un periodo de cinco años consecutivos no se sobrepasen los 100 miliSieven y nunca los 50 miliSieven en un solo año; 2) para manos, pies y piel será de 500 milisieven y 3) para el cristalino de 150 miliSieven.

En el cuadro I se muestran algunos límites de exposición (Sv) y su efecto biológico probable (Greenberg, 2009).

Durante el manejo de materiales radiactivos se requiere aplicar principios y regulaciones con el fin de proteger la salud de las personas y el medio ambiente (Ringius, 200I, SEPR, 2002). Cualquier organización que esté directa $\mathrm{O}$ indirectamente asociada con materiales radiactivos debe establecer los criterios tendientes a proteger la salud de la población de los riesgos radiológicos que puedan derivarse del uso de radiaciones ionizantes y actividades afines (OECD, 20 I0, OIEA, 2003, University of Liverpool, 2008).

En Costa Rica se utilizan materiales radiactivos (fuentes selladas), así como compuestos químicos e insumos, en el marco de los usos pacíficos de la energía nuclear. En el cuadro 2 se muestran algunas de las fuentes utilizadas en el país (Estado de la Nación, 2004).

Además, el país utiliza compuestos químicos radiactivos como el citrato de galio y el óxido de torio. En los hospitales, el medicamento radiactivo más utilizado es el yoduro de sodio-131, cuyo periodo de semidesintegración es de 8,04 horas. 
Cuadro I. Límites de exposición general y efectos biológicos de las radiaciones.

\begin{tabular}{|c|c|}
\hline Dosis agudas (Sv) & Efecto probable \\
\hline $0-25$ & Ninguna lesión evidente. \\
\hline $25-50$ & Posibles alteraciones en la sangre pero ninguna lesión grave. \\
\hline $500-100$ & Alteraciones de las células sanguíneas. Alguna lesión. Ninguna incapacitación. \\
\hline $100-200$ & Lesión. Posible incapacitación \\
\hline 200 & Certeza de lesión e incapacitación. Probabilidad de defunción. \\
\hline 400 & $50 \%$ de mortalidad. \\
\hline 600 o más & Mortal. \\
\hline
\end{tabular}

Cuadro 2. Fuentes radiactivas utilizadas en Costa Rica.

\begin{tabular}{|c|c|c|}
\hline Fuente radiactiva & Uso & Vida media \\
\hline Cobalto-60 & Servicios de radioterapia & 5.27 años \\
\hline Yodo-125 & Laboratorios de radioinmunoanálisis & 60.1 días \\
\hline Tecnesio-99m & Servicios de medicina nuclear & 6.02 horas \\
\hline Iridio-192 & Radiografías, Gammagrafías & 30 años \\
\hline Cesio-137 & Industria (control de calidad) & 30 años \\
\hline Americio-Berilio 242 & Industria (control de calidad) & 30 años \\
\hline Estroncio-90 & Industria (control de calidad) & 29.1 años \\
\hline
\end{tabular}

Las cantidades que ingresan al país dependen de las necesidades que tienen las empresas, universidades - establecimientos médicos. En total hay 270 fuentes que se usan con fines médicos y 43 fuentes en empresas (Estado de la Nación, 2004). En el caso específico de instituciones de enseñanza superior y por la variedad de sus actividades, se utilizan materiales radiactivos (fuentes selladas y radioisotopos) que generan residuos radiactivos, como líquidos (agua, centello, alcoholes, cetonas y ácidos, entre otros) y sólidos (material metálico punzocortante, papel, algodón, excretas, cadáveres, bolsas, guantes y cristalería, entre otros) contaminado con material radiactivo (Biedscheid \& Devarakonda, 2005, McLintock, 1996, Mora \& Varela, 2006).

Respecto a los materiales radiactivos utilizados en las universidades, en Costa Rica se utilizan fuentes desde baja a muy alta toxicidad, tal y como se indica en el cuadro 3 (Mora \& Varela, 2006)
En Costa Rica, la legislación indica que en las instalaciones donde se empleen fuentes o material radiactivo debe existir un lugar especialmente diseñado para el almacenamiento temporal de sus desechos, debidamente autorizado por la autoridad competente. Además, deben contar con un plan de manejo de desechos radiactivos durante todas sus etapas: identificación, segregación, almacenamiento, uso y disposición final (Decreto Ejecutivo № 24037S). No obstante, a pesar de la reglamentación existente, la gestión de residuos radiactivos no ha sido debidamente abarcada y no existe formalmente un plan nacional de manejo de desechos radiactivos, lo que limita su gestión en las distintas instituciones y por ende el tratamiento y disposición final de los residuos (Estado de la Nación, 2004).

El objetivo principal de la presente investigación es identificar los aspectos administrativos, técnicos y operativos relacionados con la gestión de productos 
Cuadro 2. Algunas fuentes radiactivas y sus radionucleidos utilizados en centros universitarios de Costa Rica.

\begin{tabular}{|c|c|}
\hline Radiotoxicidad & Radionucleidos \\
\hline Muy alta & $\mathrm{Am}^{241}, \mathrm{Ra}^{226}, \mathrm{Cm}^{244}, \mathrm{Pu}^{239}, \mathrm{Cf}^{252}$ \\
\hline Alta & $\mathrm{Mn}^{54}, \mathrm{Na}^{22}, \mathrm{Sr}^{90}, \mathrm{Co}^{60}, \mathrm{Cs}^{137}, \mathrm{Cl}^{136}, \mathrm{Bi}^{210}$ \\
\hline Moderada & $\mathrm{Zn}^{65}, \mathrm{Fe}^{55}, \mathrm{Cd}^{109}, \mathrm{Eu}^{152}, \mathrm{Co}^{57}, \mathrm{Sn}^{113}, \mathrm{C}^{14}, \mathrm{Tc}^{99}, \mathrm{Y}^{90}, \mathrm{Pm}^{147}$ \\
\hline Baja & $\mathrm{Th} 232, \mathrm{U}^{138}, \mathrm{U}_{3} \mathrm{O}_{88}, \mathrm{UO}_{2}$ \\
\hline
\end{tabular}

y residuos radiactivos que realiza el Laboratorio de Isotopos de la Universidad de Tübingen en Alemania, entidad responsable de la gestión de materiales radiactivos de esta institución.

\section{Metodología}

Sitio de Investigación

Este trabajo de investigación se realizó en el Laboratorio de Isotopos y Protección Radiológica de la Universidad de Tübingen, Alemania, del I de abril al 3 de junio de 20/3. El laboratorio de isotopos y protección radiológica de esta universidad fue creado en 1975, con el fin de brindar apoyo a todas las instancias de la universidad relacionadas con el uso de materiales radiactivos, cumpliendo de esta manera con la normativa nacional e internacional y procurando así la protección de la salud de profesores, estudiantes y empleados en general, así como del medio ambiente. El laboratorio cuenta con I 5 personas para cumplir con sus labores administrativas y técnico-operativas, desarrollando sus actividades en las siguientes áreas internas ( $w$ ww. uni-tuebingen.de/isotopenlabor):

- Bodega de productos radiactivos y fuentes selladas.

- Tres laboratorios de control de calidad (análisis de reactividad en muestras de suelo, agua, material sólido y líquido, medicamentos, materia prima).

- Cuarto instrumental de medición de radiactividad.

- Cuarto instrumental para la protección radiológica.

- Zona de almacenamiento temporal y permanente de residuos radiactivos.
- Vehículo para transporte de material radiactivo.

- Áreas administrativas.

- Salones de reunión y capacitación.

Entre los servicios que brinda el laboratorio están: capacitación en el uso de materiales radiactivos, entrenamiento a estudiantes e investigadores, dotación de licencias a personas para el uso de materiales radiactivos, control de la exposición (mediante dosimetría) en personas expuestas directamente a materiales radiactivos, transporte de materiales y residuos y administración de la protección radiológica.

\section{Generación de información}

Con el fin de identificar los procedimientos administrativos y técnico-operativos relacionados con el uso, almacenamiento, transporte y disposición final de los materiales radiactivos utilizados en el sitio de investigación, se realizaron las siguientes actividades: I) desarrollo de un cuestionario y/o lista de chequeo, 2) visita (inspección) y aplicación de los cuestionarios a los sitios del laboratorio donde se usan y almacenan materiales radiactivos, 3 ) sesiones de trabajo con funcionarios y 4) revisión de planes, protocolos, procedimientos y directrices.

Análisis de datos

La información obtenida a partir de la aplicación de los cuestionarios, entrevistas, visitas y la documentación investigada se recopiló y analizó con el fin de identificar, analizar y actualizar las actividades administrativas y técnico- operativas realizadas por el Laboratorio de Isotopos y Protección Radiológica de la Universidad de Tübingen. Se identifico y analizó la gestión de materiales radiactivos en relación con los siguientes dos aspectos: I. Administrativos (manuales y protocolos para la protección radiológica, normativas internas para manejo de material 
radiactivo); y 2. Técnico-operativos (actividades para el control de la contaminación, infraestructura, equipos y materiales de seguridad para la medición, control y seguimiento de la radiación, tratamiento y disposición final de residuos radiactivos).

\section{Resultados y discusión}

\section{Aspectos administrativos}

Entre las acciones que el laboratorio gestiona respecto a la protección radiológica están las siguientes: definir áreas de acceso a sitios que manipulan materiales radiactivos, brindar información para acceso a áreas restringidas, brindar datos de contacto (nombre, teléfonos) en caso de emergencias, definir y dotar de licencias a personas para que trabajen con materiales radiactivos, controlar la radiación física y médica, definir acciones para la importación y exportación de materiales radiactivos, definir medidas de protección radiológica para fuentes selladas y no selladas, monitorear y supervisar la contaminación radiológica, habilitar áreas para el trabajo con materiales radiactivos, coordinar la operación de equipo médico, físico, químico, etc. relacionado con fuentes radiactivas y hacer la medición de las tasas de dosis y de exposición de las personas, entre otros. Con el fin de garantizar el cumplimiento de estas acciones, el laboratorio genera y aplica diferentes protocolos, manuales y normativas para la gestión adecuada de materiales y residuos radiactivos.

\section{Protocolos}

Entre los protocolos más relevante están los siguientes: I) Formulario para el manifiesto de residuos radiactivos, 2) Procedimiento para la disposición de materiales radiactivos en el ambiente, 3) Protocolo para la manipulación de materiales radiactivos por encima del límite permitido, 4) Formulario para la recolección de materiales radiactivos, 5) Procedimiento para la inscripción de personal para protección radiológica, 6) Procedimiento para la manipulación de materiales radiactivos, 7) Procedimiento para la aplicación de capacitaciones iniciales, 8) Formulario de examen médico para la protección radiológica, 9) Formulario para el registro de la dosimetría, I0) Protocolo para la solicitud de trabajo en el laboratorio de isotopos y protección radiológica , II) Directriz para la entrega de productos radiactivos, 12) Protocolo de solicitud de sustancias radiactivas.
Manuales

El manual más importante es el Manual para la Protección contra la Radiación en la Universidad de Tübingen, creado en 200I por el laboratorio con el fin de brindar toda la información necesaria a la población universitaria y externa (visitantes, proveedores, etc.). Este manual contiene guías específicas e instrucciones para el personal de operación (personas que se exponen diaria y directamente con materiales radiactivos), de limpieza, de mantenimiento y operación, usuarios, visitantes, profesores y estudiantes. El manual indica todos los procedimientos para el uso, manejo, disposición y transporte de materiales radiactivos tanto para la parte operativa como para el entorno administrativo. Asimismo, indica lo que se debe hacer en caso de situaciones de emergencia, el personal de contacto, etc. Con este manual, el laboratorio busca mantener un ambiente de trabajo sano dentro de sus instalaciones y sobre todo brindar información de primera mano al usuario para una adecuada protección radiológica.

\section{Normativa}

Parte de la documentación que elabora y aplica el laboratorio es resultado de la normativa nacional e internacional vigente. En este sentido, el laboratorio genera y cumple con la siguiente normativa: Ley de Energía Atómica, Directiva Europea 96/29 Euratom, Reglamento para la protección de radiaciones ionizantes, Reglamento administrativo general, Directrices relativas para los conocimientos técnicos en materia de protección radiológica, Directivas para prueba de fugas en materiales radiactivos cerrados, Directiva para la vigilancia de la protección radiológica (física) para identificar dosis corporales, Reglamento de protección contra el daño causado por los rayos X y Aplicación de la Ordenanza de Protección contra la Radiación.

\section{Aspectos técnico-operativos}

Las siguientes son las actividades y acciones desarrolladas dentro del laboratorio para el cumplimiento de la gestión técnico-operativa.

Actividades para el control de la contaminación

Infraestructura, equipos y materiales

de seguridad para la medición, control

y seguimiento de la radiación

El Laboratorio de Isotopos y Protección Radiológica de la Universidad de Tübingen cuenta con sitios 
para el almacenamiento y tratamiento de materiales radiactivos, así como laboratorios donde tanto el personal interno como el externo pueden realizar diferentes actividades relacionadas con el control de la contaminación. El cuadro 3 muestra parte de estas facilidades y acciones.

Tratamiento y disposición final de residuos radiactivos

Con respecto al manejo de los residuos radiactivos generados en la universidad, el personal del laboratorio lleva un control estricto tanto de los desechos generados en sus propias instalaciones como en sitios externos, cumpliendo con todas las etapas de un adecuado sistema de gestión de residuos (identificación, segregación, transporte, almacenamiento y disposición final).

Cada ente generador es el responsable único de identificar correctamente los recipientes donde almacenan sus residuos e identificar su contenido. Para la identificación del residuo, el generador debe utilizar el formulario correspondiente que el laboratorio ha diseñado y distribuido con antelación. En el formulario se debe indicar, entre otros datos, los isotopos y su actividad radiactiva aproximada.

Una vez que los residuos radiactivos son recibidos en el laboratorio, se verifica la actividad radiactiva declarada por el generador. Para los residuos líquidos se toma una muestra en un vial y se analiza su actividad en un contador electrónico. Para los residuos sólidos se utiliza un detector portátil con el fin de obtener un dato previo y se corrobora la actividad radiactiva mediante su medición en un contador electrónico.

Posteriormente a la medición de la actividad radiactiva, los residuos tanto sólidos como líquidos son almacenados hasta que su radiactividad esté dentro de los límites permisibles para ser dispuestos como residuos ordinarios (no peligrosos) o recibir un tratamiento externo como residuos peligrosos no radiactivos. La actividad radiactiva de los residuos almacenados (sólidos y líquidos) se mide frecuentemente dependiendo de la vida media de cada isótopo (horas, días, meses, años).

Cuando la radiactividad de los residuos sólidos está por debajo del límite permitido, se dispone de ellos como basura ordinaria. En caso de que los residuos sólidos presenten actividad radiactiva (superior a los estándares permisibles) se almacenan en un sitio acondicionado para tal fin hasta que su actividad decaiga y posteriormente se dispone de ellos como residuos ordinarios.

Los residuos líquidos pueden ser tanto acuosos como orgánicos. Si ambos tipos de residuos tiene una actividad radiactiva permisible para ser tratados externamente, se procede de la siguiente manera: a) los residuos acuosos son descargados al sistema de tratamiento de aguas residuales de la universidad, y b) los residuos orgánicos son tratados mediante una planta de incineración externa. Los residuos acuosos son principalmente aguas de lavado de los diferentes sitios de la universidad que trabajan con material radiactivo; estos se transportan mediante tuberías hasta tanques de almacenamiento ubicados dentro del laboratorio, donde se les mide diariamente su radiactividad. En caso de que los residuos orgánicos presenten una actividad por encima del límite permisible, se deben almacenar temporalmente en el laboratorio hasta que ésta decaiga. Los residuos acuosos se mantienen en los tanques de almacenamiento hasta que su radiactividad permita el descargue al sistema de alcantarillado de la universidad. La figura I muestra el flujo de las actividades en el tratamiento de los residuos radiactivos sólidos y líquidos acuosos y líquidos orgánicos.

\section{Conclusiones}

Se identificaron y analizaron los aspectos administrativos y técnicos-operativos realizados en el Laboratorio de Isotopos y Protección radiológica de la Universidad de Tübingen para la gestión de materiales radiactivos. Entre los aspectos administrativos se identificaron protocolos, manuales y normativas generados en el laboratorio que son objeto de aplicación institucional. Los protocolos permiten acciones como: manifestación de residuos radiactivos, disposición de materiales radiactivos en el ambiente, manipulación de materiales radiactivos por encima del límite permitido, recolección de materiales radiactivos e inscripción de personal para protección radiológica.

El manual más importante es el Manual para la Protección contra la Radiación en la Universidad de Tübingen, que brinda toda la información necesaria a la población universitaria y externa (visitantes, proveedores, etc.) para la protección radiológica. 
Cuadro 3. Facilidades para la gestión de materiales radiactivos del Laboratorio de Isotopos y Protección Radiológica de la Universidad de Tübingen.

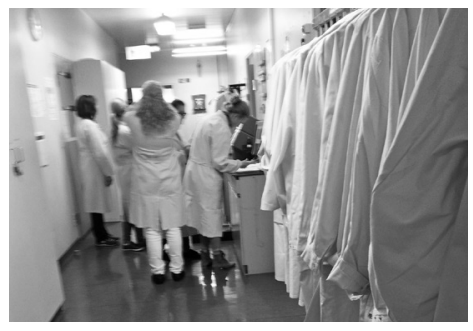

Zona de gabachas: Cada persona que trabaja dentro del laboratorio tiene gabachas asignadas y existen gabachas para los visitantes.

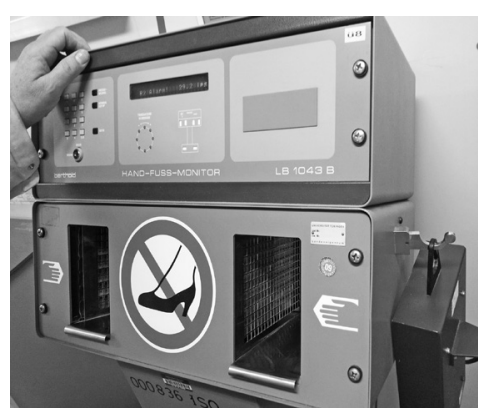

Equipo para verificación de la contaminación personal.

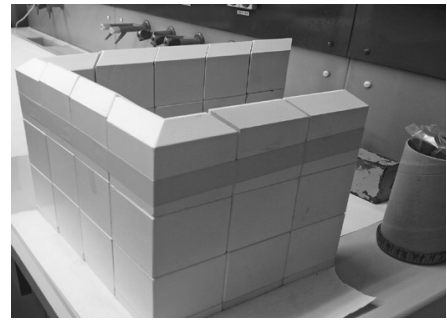

Zona de trabajo con material radiactivo; el compartimiento es un material que minimiza la exposición a la radiación mientras la persona trabaja.
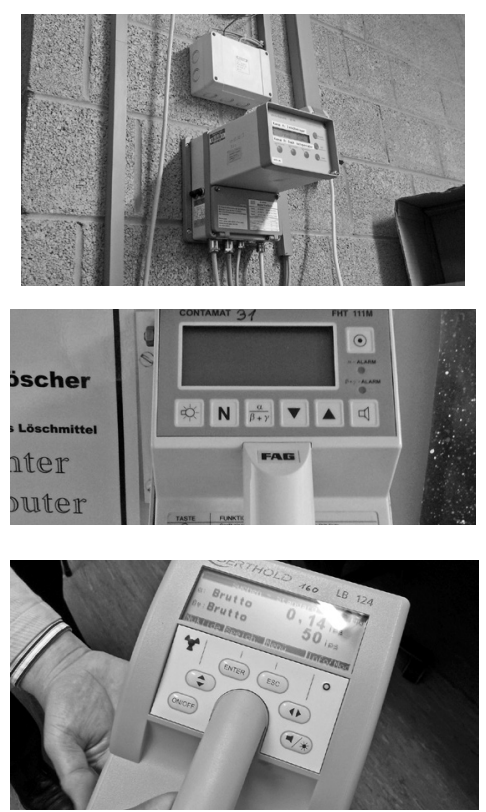

Equipos portátiles y fijos (dosímetros, medidores y detectores de radiación) utilizados para medir la contaminación o emisión radiactiva de diferentes materiales.

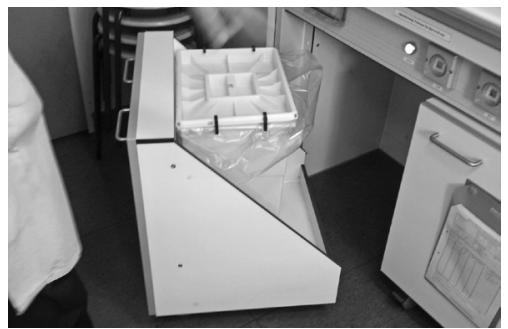

Capillas de extracción y gabinetes especiales para almacenar material radiactivo.

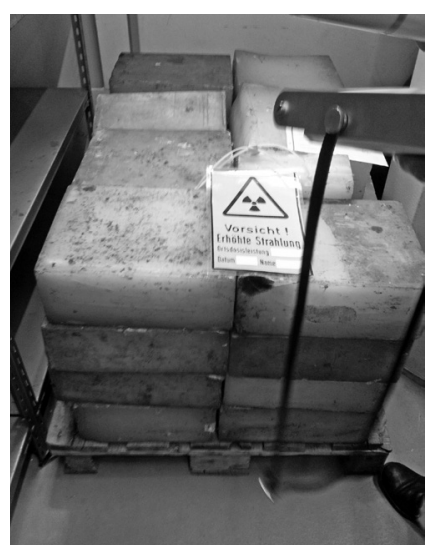

Almacenamiento de materiales radiactivos con alta actividad; se utiliza un recubrimiento externo con parafina y en la parte interna láminas de plomo.
El laboratorio cumple y genera normativa relacionada con la Ley de Energía Atómica, la Directiva Europea 96/29 Euratom, el Reglamento para la protección de radiaciones ionizantes y la Directiva para la vigilancia de la protección radiológica (física) para identificar dosis corporales.

Respecto a los aspectos técnicos operativos, se identificaron acciones y disponibilidad de infraestructura, equipos y materiales para la medición y el control de la radiación entre los que se destaca el uso de dosímetros, medidores y detectores de radiación, zonas de trabajo para material radiactivo, sitios para almacenamiento de material radiactivo, capillas para uso exclusivo de material radiactivo.

Finalmente, se analizó el tratamiento y disposición final de los residuos sólidos y líquidos contaminados con material radiactivo. Se constató que el personal del laboratorio lleva un control estricto tanto de los residuos generados en sus propias instalaciones como de los provenientes de sitios externos, 


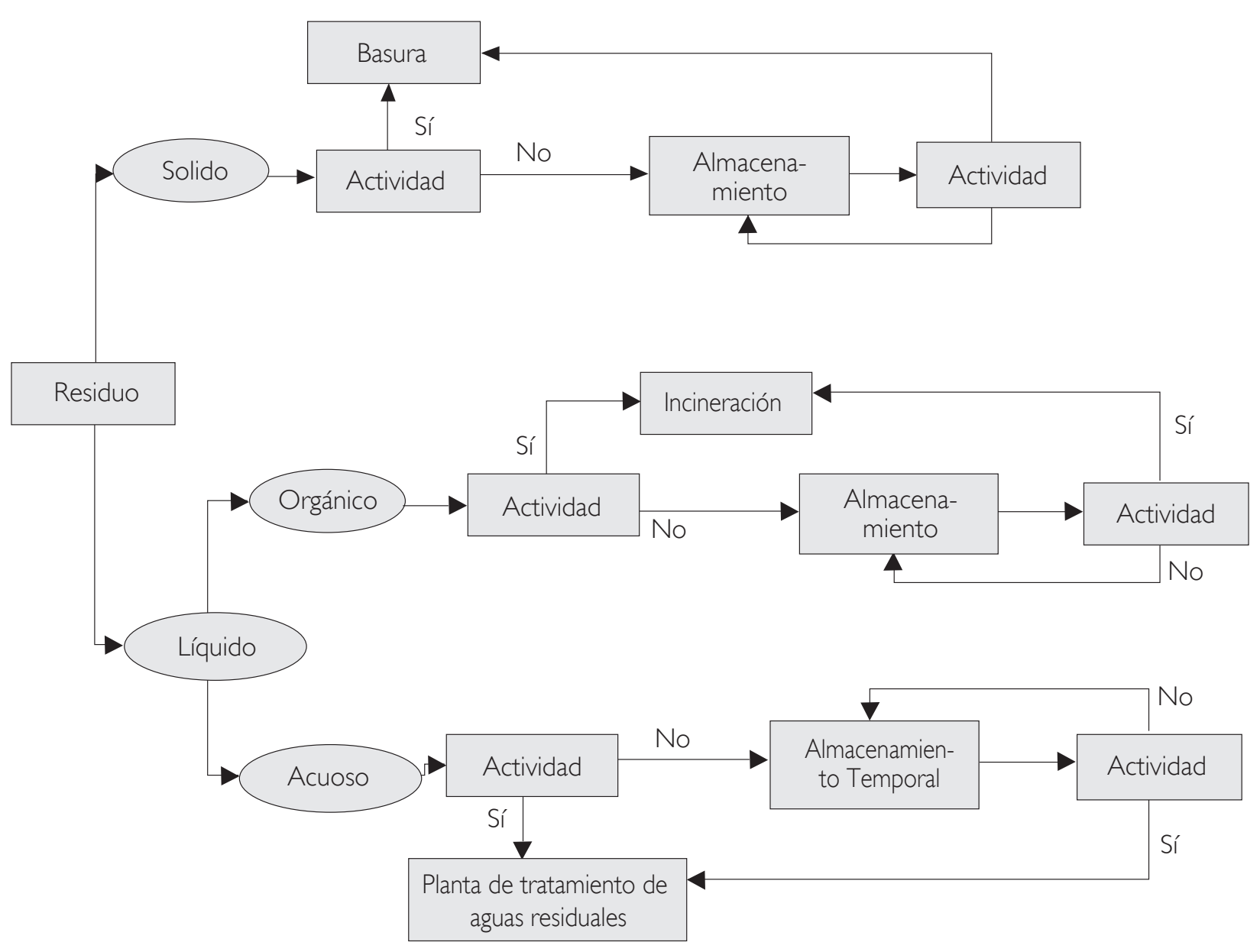

Figura I. Flujograma de la gestión de residuos radiactivos en el Laboratorio de Isotopos y Protección Radiológica de la Universidad de Tübingen, Alemania.

cumpliendo con todas las etapas de un adecuado sistema de gestión (identificación, segregación, transporte, almacenamiento y disposición final).

Como conclusión principal se debe indicar que durante la gestión de materiales radiactivos es imprescindible el cumplimiento de la normativa internacional, esencialmente de las directrices de la OIEA. Esta normativa debe ser adaptada al quehacer universitario mediante procedimientos, manuales y reglamentos internos para que sea de conocimiento y aplicación del personal relacionado con la gestión de materiales radiactivos, tal y como lo hace el Laboratorio de Isotopos y Protección Radiológica de la Universidad de Tübingen.

Los datos obtenidos como resultado de la investigación realizada constituyen una herramienta de gran utilidad para generar y mejorar propuestas para el uso adecuado de materiales y residuos radiactivos en centros universitarios, de manera que sirvan como base para otras entidades académicas e instituciones públicas y privadas.

\section{Agradecimientos}

Los autores agradecen al Laboratorio de Isotopos y Protección Radiológica de la Universidad deTübingen, Alemania, la Junta de Becas, la Universidad Nacional y la Agencia Académica de Intercambio Alemán (German Deutscher Akademischer Austauschdienst).

\section{Bibliografía}

Alexander,W. \& McKinley, L.E. (2007). Deep geological disposal of radioactive waste. Amsterdam: Elsevier.

Berlin, R. \& Stanton, C. (1989). Radioactive waste management. New York: Wiley. 
Berlin, R. \& Stanton, C. (1989). Radioactive waste management. New York: Wiley.

Biedscheid, J, \& Devarakonda, M. (2005). Radioactive waste. Reston, Va.,: American Society of Civil Engineers.

Decreto Ejecutivo 24037-S. (1995). Reglamento Sobre Protección Contra las Radiaciones. San José: La Gaceta.

DSRL (Dirección del Servicio de Riesgos Laborales). (2009). Procedimientos de protección radiológica de fuentes no encapsuladas utilizadas en la instalación radiactiva central de la Facultad de Medicina de la Universidad Complutense de Madrid. Universidad Complutense de Madrid.

Estado de la Nación (2004). Programa Estado de la Nación en Desarrollo Humano Sostenible. Resumen del Undécimo Informe Estado de la Nación en Desarrollo Humano Sostenible. San José.

Estado de la Nación (2007). Plan Nacional de Residuos Sólidos de Costa Rica, Diagnóstico y Áreas Prioritarias. San José.

Greenberg, M. (2009). The reporter's handbook on nuclear materials, energy, and waste management. Vanderbilt University Press.

McCutcheon, C. (2002). Nuclear reactions: the politics of opening a radioactive waste disposal site. University of New Mexico Press.

McLintock, S. (1996). The management of radioactive waste in laboratories. Leeds, England: $\mathrm{H}$ and $\mathrm{H}$ Scientific Consultants.

Mora, P. \& Varela, A. (2006). Manejo responsable de los desechos radioactivos en la Universidad de Costa Rica. Revista Ciencia y Tecnología 24( I): 9-20.

OECD (Nuclear Energy Agency, Organisation for Economic Co-operation and Development) (2010). Radioactive waste in perspective. Paris.
OIEA (Organismo Internacional de Energía Atómica). (2009). Gestión de desechos procedentes de la utilización de materiales radiactivos en medicina, industria, agricultura, investigación y educación. Colección de normas de seguridad del OlEA. Viena.

OIEA (Organismo Internacional de Energía Atómica). (2003). Predisposal Management of Low and Intermediate Level Radioactive Waste. Colección de Normas de Seguridad del OIEA, Viena.

Ringius, L. (200I). Radioactive waste disposal at sea: public ideas, transnational policy entrepreneurs, and environmental regimes. Cambridge, Massachusess, US: MIT Press.

Schleien, B. (1992). Nuclear Transportation Including Shipment of Low-Level Waste. The Health Physics and Radiological Health Handbook. Revised Edition, Chapter 14. Silver Spring MD, US: Scinta, Inc.

SEPR (Sociedad Española de Protección Radiológica). (2002). Guía técnica de gestión de materiales residuales con contenido radiactivo en centros de investigación y docencia. Madrid: Sociedad Española de Protección Radiológica.

Simon, R. (2009). Radioactive waste management and disposal. Cambridge University Press.

http://www.uni-tuebingen.de/en/university.html. Consultado en junio de 2013.

University of Liverpool. (2008). Management of radioactive waste within the University of Liverpool. England: University of Liverpool.

Vandenbosch, R \& Vandenbosch, S. (2007). Nuclear waste stalemate: political and scientific controversies. University of Utah Press.

www.uni-tuebingen.de/isotopenlabor Consultado en junio de 2013. 